\title{
A Combined Mathematical-Physical Model of Laser-Induced Thermotherapy (LITT)
}

\section{Enevoldsen, Marie Sand; Skovgaard, Ove; Andersen, Peter E.}

Published in:

Therapeutic Laser Applications and Laser-Tissue Interactions IV

Link to article, DOI:

$10.1117 / 12.831933$

Publication date:

2009

Document Version

Publisher's PDF, also known as Version of record

Link back to DTU Orbit

\section{Citation (APA):}

Enevoldsen, M. S., Skovgaard, O., \& Andersen, P. E. (2009). A Combined Mathematical-Physical Model of Laser-Induced Thermotherapy (LITT). In Therapeutic Laser Applications and Laser-Tissue Interactions IV: Proceedings of SPIE (Vol. Vol. 7373) https://doi.org/10.1117/12.831933

\section{General rights}

Copyright and moral rights for the publications made accessible in the public portal are retained by the authors and/or other copyright owners and it is a condition of accessing publications that users recognise and abide by the legal requirements associated with these rights.

- Users may download and print one copy of any publication from the public portal for the purpose of private study or research.

- You may not further distribute the material or use it for any profit-making activity or commercial gain

- You may freely distribute the URL identifying the publication in the public portal 


\title{
A Combined Mathematical-Physical Model of Laser-Induced Thermotherapy (LITT)
}

\author{
Marie S. Enevoldsen ${ }^{a}$, Ove Skovgaard ${ }^{b}$ and Peter E. Andersen ${ }^{c}$ \\ ${ }^{a}$ DTU Electrical Engineering, Oersteds Plads Build. 349, Kgs. Lyngby, Denmark; \\ ${ }^{b}$ DTU Mathematics, Matematiktorvet Build. 303S, Kgs. Lyngby, Denmark; \\ ${ }^{c}$ DTU Fotonik, Frederiksborgvej 399 Build. OPL-108, Roskilde, Denmark
}

\begin{abstract}
Laser-induced thermo therapy (LITT) is an alternative, gentle therapy of cancer. In this work a new computational model (3D space and time) of LITT is presented. Using an arbitrary small number $(<20)$ of optical fibers, multiple low energy laser light sources are applied internal to an arbitrary shaped tumor in the human liver. The power and position of each source can be chosen arbitrary. Each source is a spherical point source emitting light isotropically. The model consists of two, semi-coupled partial differential equations (PDEs) describing the light distribution and the heat absorption in the target tissue. Since water is a dominant tissue component in both the healthy liver and the malignant tumor the wavelength of the laser is chosen in the NIR area $(1,064 \mathrm{~nm})$. This is expected to form an absorption contrast in favor of the tumor leading to high temperature and damage of the tumor cells. The new, fast computational model presented here opens for the possibility of evaluating the outcome of LITT by inspection of temperature fields, and comparing these to measured histological damage due to heating. This combination is promising when evaluating the result of LITT prior to the actual treatment.
\end{abstract}

Keywords: LITT, liver metastasis, water contrast, semi-coupled PDEs

\section{INTRODUCTION}

Treatment of cancer has become top priority in health care sectors world wide and a large amount of resources are invested in improved therapy methods for the numerous cancer patients. The field of experimental therapies is growing because of the need to find therapies that both cause minimal pain and result in high mean survival rates. One of the promising new, alternative forms of therapy is laser-induced thermotherapy (LITT). With LITT irreversible damage to malignant tissue is achieved by applying low power laser light to the tissue.

The purpose of applying lasers in therapeutic procedures is to use the laser light to heat the target tissue above $50{ }^{\circ} \mathrm{C}$. This is because when the human tissue is heated above $50{ }^{\circ} \mathrm{C}$ the cells are irreversibly damaged leading to initiation of programmed cell death (apoptosis).

LITT applied on malignant, cancer tumors was first described by Bown in $1983 .{ }^{1}$ Interstitial thermal damage is achieved by employing low power laser light through thin optical fibers, see Fig. 1.

LITT is minimally invasive compared to surgical removal of a tumor, since the thin optical fibers are flexible and they can be applied directly in the tumor through small holes in the skin. In contrast to the removal of the tumor the damaged tissue is not removed from the site when using LITT. Another issue with surgical removal of malignant tumors is that not all patients or tumors are suited for this treatment. Either the patient is too ill or the tumor is situated near or in vital structures. The alternative to surgical removal is either chemotherapy or ionizing radiation. Especially chemotherapy leads to significant side effects which can be avoided by using LITT. The mean survival rates of LITT are equally high compared to surgical removal. ${ }^{2}$

Broad clinical experience with LITT is found in Sweden and Germany, but only few, general computational models of the processes in LITT have been published. Wasmer et.al. ${ }^{3}$ and Olsrud et.al. ${ }^{4}$ simulates LITT in a

Further author information: (Send correspondence to M.S.E.)

M.S.E.: E-mail: mse@elektro.dtu.dk, Telephone: +45 45253668

O.S.: E-mail: ove.skovgaard@mat.dtu.dk, Telephone: +45 45253012

P.E.A.: E-mail: peta@risoe.dtu.dk, Telephone: +45 46774555 


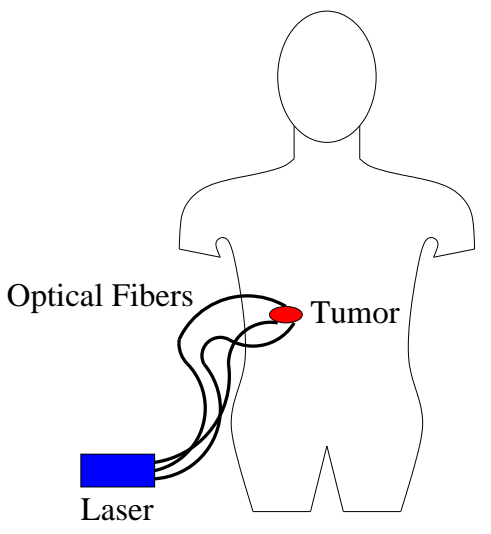

Figure 1: The principle of interstitial LITT.

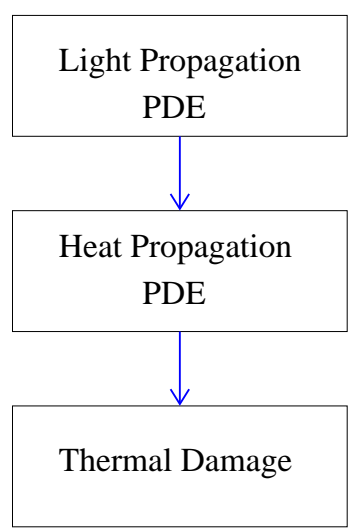

Figure 2: Elements in the model.

3D, axis symmetric, homogeneous tissue geometry with symmetric, internal placements of light sources. Feng et.al. ${ }^{5}$ uses one external light source on an arbitrary, malignant tumor.

Liver metastases are selected for modeling in this work for several reasons. The liver is the second most frequent site for metastatic spread of primary, malignant tumors. The main sources of metastases in the liver are tumors in the breast, the colon, the stomach and the lungs, which are all among the most common types of cancer. The preferred treatment of liver metastases is surgical removal of the malignant tissue, but many patients are unsuited for surgery for various reasons, for example when the metastasis is placed close to a large vein. The alternative to surgical removal is chemotherapy, but the mean survival rate is significantly lower compared to removal. ${ }^{2}$ Ionizing radiation is not considered for liver metastases. LITT is documented to be an effective therapy for liver metastases. ${ }^{6}$ Furthermore, the liver is large and the tissue is fairly uniform. This results in a model with only two different tissue types, malignant and benign, and this simplifies the general model.

The motivation of this work is to visualize the outcome of LITT through fast computational simulation of LITT in a general model. The computational simulation of LITT needs to be applicable to any type of cancerous tumor of arbitrary shape, and the number of laser light sources should be optional. Furthermore, a successful outcome of LITT is based on proper guiding and accurate placement of the thin optical fibers.

This work presents a new, general model of LITT using semi-coupled partial differential equations (PDEs) for the distribution of light energy and resulting heat distribution, see Fig. 2. The PDEs are dependent in both time and 3D space, and it is possible to apply an arbitrary number of internal light sources at arbitrary positions in the model volume. Furthermore, the model is not symmetric and includes two types of tissue, healthy and cancerous, and due to reasonable simplifying assumptions the computation time is kept low.

\section{MATERIALS AND METHODS}

The computational model is constructed in COMSOL Multiphysics vers. 3.4 (1994-2007 COMSOL AB) a commercial software for solving PDEs by the finite element method.

One liver metastasis of arbitrary shape and realistic size is chosen for modeling, see Fig. 3. The model example in this work is a box of healthy liver surrounding the metastasis in the center. The box is large enough to assume simple boundary conditions for the semi-coupled PDEs, and at the same time small enough to only comprise a part of the liver. Both the liver and metastatic tissues are assumed to be homogeneous, thus only the optical and thermo-physiological properties vary between the two tissue types. By choosing the modeling volume to be only a part of the liver and large enough to be valid for the applied boundary conditions, it is possible to keep the model geometry fairly simple. 


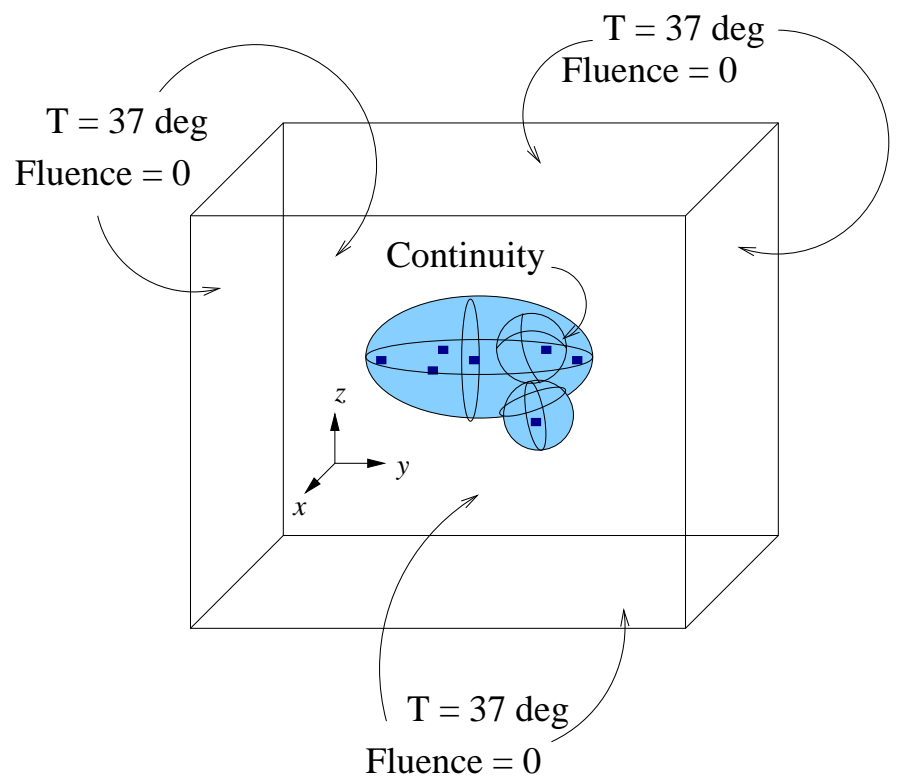

Figure 3: Geometry and boundary conditions (BC) of the model. The light sources are indicated with squared points.

Table 1: Mesh settings of the finite element model.

\begin{tabular}{|l||c|c|}
\hline Setting & Liver & Metastase \\
\hline \hline No. elements & 22,867 & 11,388 \\
No. sources & 0 & 7 \\
\hline
\end{tabular}

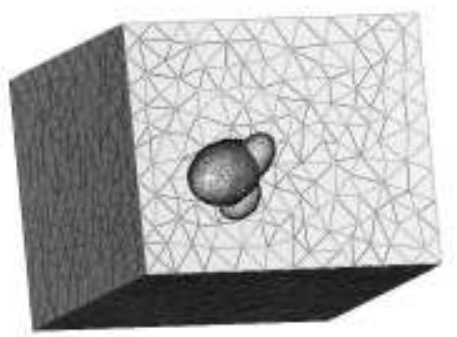

Figure 4: Finite elements of the model.

Due to the simple representation of the model geometry and the light sources, the computation time for the model is kept low when using an iterative conjugate gradients solution method of the algebraic equations in the FEM model. The model settings of the finite element model are displayed in Table 1 and Fig. 4.

\subsection{Tissue Optics}

When modeling light propagation in tissue it is important to assign the influence from the optical properties. Multiple scattering is dominating the light propagation in both tissue types, but it is the absorption of the laser light in the tissue that trigger the temperature rise needed for killing the cancer cells.

\subsubsection{Choice of Wavelength}

Characterization of the composition of chromophores* in the liver and the metastasis is essential for determining a suitable wavelength for the applied laser in LITT. With a suitable wavelength it is expected that an absorption contrast in the malignant tissue compared to the normal, healthy liver tissue, see Fig. 5 because more light is absorbed in the metastasis. The contrast can be established because the tissue types consists of different chromophores with different absorption spectra. In the two tissue types there are three dominating chomophores; protein, hemoglobin and water.

${ }^{*}$ Light absorbing particles in the tissue. 
For protein to dominate the light absorption the wavelength should be in the ultraviolet (UV) area of the electromagnetic spectrum. But scattering is expected to be too dominating due to the short wavelength, thus choosing a wavelength in the UV area is not expected to give a successful outcome of LITT.

Hemoglobin is the oxygen carrying part of the red blood cells. Since the blood is moving through the liver at a high volume flow rate, the heat resulting from the absorbed light is not expected to be focused in the target tissue. Therefore, choosing a wavelength in the area where the hemoglobin is the dominating chromophore is not expected to yield an optimal treatment with LITT.

Water is a dominant component in both liver (60-70\%) and metastatic tissue $(80-90 \%)^{7}$ and has several absorption peaks in the electromagnetic spectrum, mainly in the near infrared (NIR) area of the electromagnetic spectrum. In this case 1,064 $\mathrm{nm}$ is choosen as wavelength because the penetration depth is high, and the light is transmitted through thin optical fibers without too much effect loss.

\subsubsection{Diffusion Model Approximation}

Analytical solutions can be found for the light propagation in a material by solving Maxwell's equations, and the solutions will include all physical effects and yield an exact description of the propagation. But in this case, where the ultimate goal of simulation of LITT is to use it in a clinical setting, finding analytical solutions is impossible, therefore numerical methods are needed.

For the numerical method the diffusion model approximation is applied to identify the propagation of light in the tissue by calculating the fluence rate, $\Phi(\mathbf{r}, t)\left[\mathrm{W}\left(\mathrm{m}^{-2}\right)\right]$, which make up the source term in the Bioheat equation, see Sec. 2.2. The diffusion model approximation is based on the time dependent transport equation (see for example W. M. Star ${ }^{8}$ ) and the source term for an isotropic point source: ${ }^{9}$

$$
\frac{1}{c} \frac{\partial \Phi(\mathbf{r}, t)}{\partial t}=-\mu_{\mathrm{a}} \Phi(\mathbf{r}, t)+\nabla \cdot(D \nabla \Phi(\mathbf{r}, t))+P_{1} \delta(\mathbf{r})+P_{2} \delta(\mathbf{r})+P_{3} \delta(\mathbf{r})+\ldots,
$$

where $c\left[\mathrm{~m}\left(\mathrm{~s}^{-1} 9\right]\right.$ is the speed of light in the material, $\mathbf{r}=(x, y, z)$ is the position in space, $t[\mathrm{~s}]$ is time, $\mu_{\mathrm{a}}\left[\mathrm{m}^{-1}\right]$ is the absorption coefficient of the tissue, $D[\mathrm{~m}]$ is the diffusion coefficient for the tissue, $P_{\mathrm{n}}[\mathrm{W}]$ is the power of each of the 'n' applied light sources, and $\delta(\mathbf{r})$ is the Dirac function. Thus, for each light source applied in the model a term is added on the right hand side in Eq. (1).

The optical properties of the two different tissue types are expressed through the absorption coefficient, the scattering coefficient, $\mu_{\mathrm{s}}\left[\mathrm{m}^{-1}\right]$, and the anisotropic factor $g$.

The similarity principle,

$$
\mu_{\mathrm{s}}^{[1]}\left(1-g^{[1]}\right)=\mu_{\mathrm{s}}^{[2]}\left(1-g^{[2]}\right),
$$

applies ( 1 and 2 indicates different values for $\mu_{\mathrm{s}}$ and $g$ ) in this case because the albedo is high in both tissue types. When the similarity principle applies the number of optical properties can be reduced by considering $\mu_{\mathrm{s}}$ and $g$ as one parameter, the reduced scattering coefficient $\mu_{\mathrm{s}}^{\prime}=\mu_{\mathrm{s}}(1-g)$. Additionally, $g$ in biological tissue is close to unity making exact measurement of $g$ difficult. Instead the $\mu_{\mathrm{s}}^{\prime}$ is measured and then used to give an estimate of $g$.

The diffusion coefficient is dependent on where the light sources are placed in the model because $D$ is calculated as

$$
D=\frac{1}{3\left(\mu_{\mathrm{a}}+\mu_{\mathrm{s}}^{\prime}\right)} .
$$

The optical properties in Eq. (1) are assumed to be independent of both temperature and time for simplicity, see Table 2. In addition, the optical dynamics are considered to be instantaneous compared to the thermal dynamics, since the optical diffusivity in the tissue is several magnitudes higher than the thermal diffusivity in the tissue. ${ }^{10}$ Thus, Eq. (1) is solved in steady state to find $\Phi(\mathbf{r})$ :

$$
\frac{\mu_{\mathrm{a}}}{D} \Phi(\mathbf{r})-\nabla \cdot(\nabla \Phi(\mathbf{r}))=\frac{P_{1}}{D} \delta(\mathbf{r})+\frac{P_{3}}{D} \delta(\mathbf{r})+\frac{P_{3}}{D} \delta(\mathbf{r})+\ldots
$$



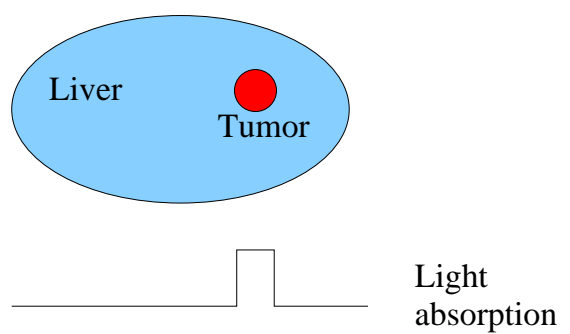

Figure 5: Principle of contrast.
Table 2: Optical properties of the tissue types involved. ${ }^{11}$

\begin{tabular}{|c||c|c|}
\hline Property & Liver & Metastase \\
\hline \hline$\mu_{a}$ & $0.05 \mathrm{~mm}^{-1}$ & $0.03 \mathrm{~mm}^{-1}$ \\
$\mu_{s}$ & $16.9 \mathrm{~mm}^{-1}$ & $10.9 \mathrm{~mm}^{-1}$ \\
$g$ & 0.952 & 0.917 \\
$\mu_{s}^{\prime}$ & $0.811 \mathrm{~mm}^{-1}$ & $0.905 \mathrm{~mm}^{-1}$ \\
\hline
\end{tabular}

\subsection{Heat Transfer in Tissue}

To derive the distribution of heat in the tissue the Bioheat equation, based on Pennes approximation, is applied in the simple model geometry. The Bioheat equation is based on an energy balance that comprises gain, loss and storage of heat in the tissue model. The properties most significant to the thermal dynamics are the thermal conductivity $k$ and the blood perfusion rate $\omega_{\mathrm{b}}$ in the liver and the metastasis. Furthermore, the specific heat, $C$, and the tissue density, $\rho$, describes the heat propagation in the tissue model. In this work the thermophysiological properties are determined by using the linear relationship between water content and the specific property, see Table 3. These properties differ significantly between the normal, healthy liver tissue and the malignant tumor tissue. Thus a marked difference in temperature is expected between the two tissue types.

Table 3: Calculated thermo-physiological properties of the liver, the metastasis and the blood. ${ }^{12,13} w$ is the water content in $\%$ in the material, and $\rho$ is the material density. The blood perfusion rate is determined from the assumption that 25 $\mathrm{mL}$ of blood flows through the liver per second.

\begin{tabular}{|c||c|c|c|c|}
\hline Property & Expression & Liver & Metastasis & Blood \\
\hline \hline$C\left[\mathrm{~J}\left(\mathrm{~kg}^{\circ} \mathrm{C}\right)^{-1}\right]$ & $1.55+2.80 \frac{w}{\rho}$ & 3,198 & 3,830 & 4,200 \\
$\rho\left[\mathrm{kg}(\mathrm{m})^{-3}\right]$ & $(1.3-0.3 w) \times 10^{3}$ & 1,105 & 1,045 & 1,050 \\
$k\left[\mathrm{~W}\left(\mathrm{~m}^{\circ} \mathrm{C}\right)^{-1}\right]$ & $0.056+0.569 \frac{w}{\rho}$ & 0.39 & 0.52 & $0.48-0.6$ \\
$\omega_{\mathrm{b}}\left[\mathrm{s}^{-1}\right]$ & - & 0.025 & 0 & - \\
\hline
\end{tabular}

The absorbed light energy described by $\Phi(\mathbf{r})$ make up the source term in the Bioheat equation:

$$
\rho C \frac{\partial T(\mathbf{r}, t)}{\partial t}=\nabla[k \nabla T(\mathbf{r}, t)]+\rho_{\mathrm{b}} C_{\mathrm{b}} \omega_{\mathrm{b}}\left(T_{\mathrm{art}}(\mathbf{r}, t)-T(\mathbf{r}, t)\right)+\mu_{\mathrm{a}} \Phi(\mathbf{r})
$$

where $T\left[{ }^{\circ} \mathrm{C}\right]$ is temperature and $T_{\text {art }}$ is the arterial temperature $\left(37^{\circ} \mathrm{C}\right)$. The index 'b' indicates property of the blood, no index indicates tissue properties.

\section{RESULTS}

The fluence rate and the temperature fields are evaluated from 3D plots, different cross sections through the box of tissue, and in specific points in the metastasis. The maximum fluence rate in the model is found in the center of the metastasis, see Fig. 6.

In Fig. 7 the result of the simulation is displayed in 3D. As can be seen, the volume of metastatic tissue is not completely destroyed after 30 minutes of irradiation because the temperature does not exceed $50{ }^{\circ} \mathrm{C}$. For a more detailed evaluation of the treatment the temporal development of temperature in three different points inside the metastasis, and the temperature field along a cross sectional line through the model is displayed in Fig. 8 and Fig. 9 respectively.

In Fig. 8 the temporal development of the temperature in three points (see Table 


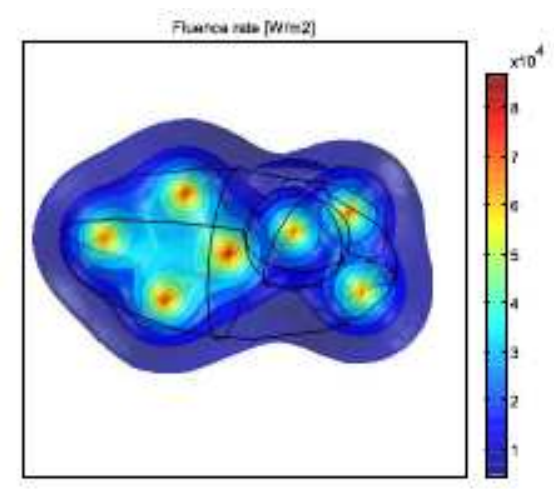

Figure 6: 3D view of the fluence rate. The position of the seven applied light sources are easily recognized.

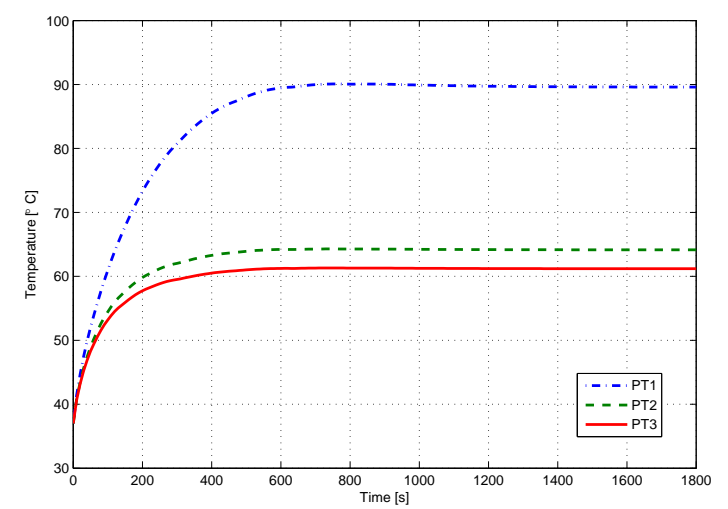

Figure 8: Temporal development of the temperature in three different points inside the metastasis, corresponding to the position of three sources, see Table ??.

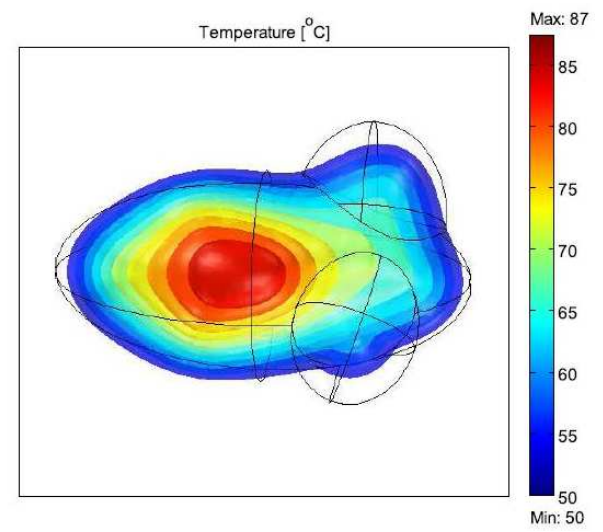

Figure 7: 3D view of the heat distribution after 30 minutes of irradiation.

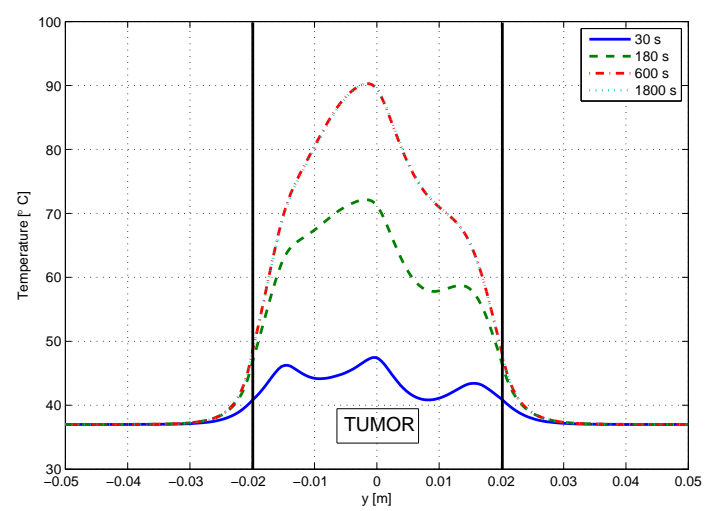

Figure 9: Temperature distribution along a straight line through the model geometry. The distribution is identical for $t=600 \mathrm{~s}$ and $t=1800 \mathrm{~s}$.

Fig. 9 shows the temperature profile along a cross section line through the center of the metastasis. The boundary of the metastasis is indicated by solid, vertical lines. The temperature inside the metastasis is above $50{ }^{\circ} \mathrm{C}$ after approximately three minutes of irradiation and the highest temperature is $90{ }^{\circ} \mathrm{C}$.

For evaluation of the result of the simulations of LITT, a criterion of success is formulated; a successful treatment is when the metastatic tissue is damaged irreversibly, and only an insignificant part of the healthy, surrounding tissue is harmed. For the target tissue to undergo irreversible damage, it is necessary to heat the tissue above $50-55{ }^{\circ} \mathrm{C}$ for a prolonged period of time. It is also important to keep the temperature below $100{ }^{\circ} \mathrm{C}$ to avoid vaporization and carbonization. Furthermore, a safety margin of a few millimeters around the metastasis should be established to secure that the entire metastasis is treated.

Thus, in this model simulation of LITT vaporization and carbonization is avoided, but no safety margin is established, see Figs. 7 and 9.

\section{DISCUSSION AND CONCLUSIONS}

The success of this work relies on well chosen simplifications. Choosing a liver metastasis as example for this work has proved to be a good decision, because it simplifies the modeling process. In addition to the modeling aspect, the liver is the second most frequent location for metastatic growth, resulting in a large group of patients suitable for LITT. Therefore the benefit of prior-treatment simulation of the outcome of LITT is expected to be 
large. This favors the use of LITT on liver metastases compared to both removal of the malignant tissue and general chemotherapy. This is because only a small group of patients is suited for operation, and the side effects of general chemotherapy are severe and should be avoided if possible.

The efficiency of LITT in this work is based on choosing the appropriate wavelength to create a contrast of energy absorption between the metastasis and the liver, in favor of the former. Water has been found to be a suitable contrast agent since water is a dominant tissue component in both the liver and the metastasis. The metastatic tissue contains more water compared to the normal liver tissue, and it is expected that more light energy is absorbed in the metastasis leading to higher temperature. The wavelength of the light is chosen to be in the NIR area of the electromagnetic spectrum $(1,064 \mathrm{~nm})$. The choice of this wavelength coincides with lasers applied in large studies. ${ }^{6}$

Comparing to other models of LITT, this work presents a general principle for modeling the outcome of LITT fast, with the possibility of applying any shape of tumor, and any number of light sources at any internal position in the tumor or the surrounding tissue. The light sources in this work are spherical point sources. Together with fast computation time, the sources can be placed to fit the target volume most optimal.

From the results of the simulation of LITT (see Figs. 6-9) by iterative solution of linear, semi-coupled PDEs, it is seen that the simulated outcome of LITT is partly successful. Several investigations of the effect of LITT indicates that it is necessary to apply several light sources to achieve a large enough damage volume to cover liver metastasis. $^{2,3}$ This corresponds with the findings in this work. This is the first step toward a tool applicable in the clinic, and therefore this is to show the principle of dynamics of this model.

The metastasis in this case is constructed with the graphical tool in COMSOL. In the clinic it will be possible to segment patient specific metastasis from magnetic resonance images and implementing these directly in the modeling process, and there by customizing the LITT to each patient to achieve the optimal outcome.

But for the customization to be effective it is necessary to establish a reliable method for determining optical and thermo-physiological properties for the liver tissue as well as the metastatic tissue. These methods are still to be established, and it is also necessary to consider the change in tissue type, because the heat affected tissue will not have the same properties as the unaffected tissue. Thus, the coefficients describing the optical and thermo--physiological properties must be time dependent so that the semi-coupled PDEs then becomes fully coupled.

Such refinements will prolong the computation time of the model. But as long as the light energy absorption is described by a PDE so the system of equations is fully coupled, it is expected that the computational time can be kept low.

\section{REFERENCES}

[1] Bown, S. G., "Phototherapy of tumors," World. J. Surg. 7, 700-709 (1983).

[2] J.-P. Ritz, C. I., Roggan, A., Buhr, H. J., and Germer, C.-T., "Laser-induced thermotherapy of liver metastases," Front. Radiat. Ther. On. 38, 106-121 (2004).

[3] Wasmer, B., Viard, R., Zemmouri, J., Rochon, P., Rousseau, J., and Mordon, S. R., "Comparison of mathematical modeling of laser-induced thermotherapy to experimental data: Determination of optimal laser parameters," in [Proceedings of the COMSOL Users Conference 2007 Grenoble], Petit, J.-M. and Squalli, O., eds., 1, 117-123 (2007).

[4] Olsrud, J., Wirestam, R., Persson, B. R. R., and Tranberg, K.-G., "Simplified treatment planning for interstitial laser thermotherapy by disregarding light transport: A numerical study," Laser. Med. Surg. 25, 304-314 (1999).

[5] Feng, Y., Fuentes, D., Hawkins, A., Bass, J., Rylander, M. N., Elliott, A., Shetty, A., Stafford, R. J., and Oden, J. T., "Nanoshell-mediated laser surgery simulation for prostate cancer treatment," Eng. Comput. 25, $3-13(2009)$.

[6] Vogl, T. J., Mack, M., Eichler, K., Lehnert, T., and Nabil, M., "Effect of laser-induced thermotherapy on liver metastases," Expert Rev. Anticancer Ther. 6, 769-774 (2006).

[7] Kiricuta, I.-O. and Simplaceanu, V., "Tissue water content and nuclear magnetic resonance in normal and tumor tissue," Cancer. Res. 35, 1164-1167 (1975). 
[8] Star, W. M., "Diffusion theory of light transport," in [Optical-Thermal Response of Laser-Irradiated Tissue], Welch, A. J. and Germert, M. J. C. V., eds., 131-205, Plenum Press, New York (1995).

[9] Esbensen, B. K. and Hoffmann, K., "Personal communications," (2009).

[10] Svaasand, L. O., "Physics of laser-induced hyperthermia," in [Optical-Thermal Response of Laser-Irradiated Tissue], Welch, A. J. and Germert, M. J. C. V., eds., 765-786, Plenum Press, New York (1995).

[11] Germer, C.-T., Roggan, A., Ritz, J. P., Isbert, C., Albrecht, D., Müller, G., and Buhr, H. J., "Optical properties of native and coagulated human liver tissue and liver metastases in the near infrared range," Laser. Surg. Med. 23, 194-203 (1998).

[12] Jacques, S. L. and Prahl, S. A., "Modeling optical and thermal distributions in tissue during laser irradiation," Laser. Surg. Med. 6, 494-503 (1987).

[13] Welch, A. J., "The thermal response of laser irradiated tissue," IEEE J. Quantum. Elect. QE-20, 1471-1481 (1984). 